\title{
Successful treatment with intraluminal fibrinogen injection of serous leakage from a polytetrafluoroethylene Blalock-Taussig shunt
}

Arata Murakami, MD, Katsuhide Maeda, MD, Hiroo Takayama, MD, Tetsuhiro Takaoka, MD, Jyotaro Kobayashi, MD, and Shinichi Takamoto, MD, Tokyo, Japan

$\mathrm{W}$ e report a case of excessive serous fluid leakage from a polytetrafluoroethylene (PTFE) BlalockTaussig shunt (BTS) that was successfully treated with intraluminal fibrinogen injection.

\section{Clinical Summary}

A 1-month-old boy weighing $3 \mathrm{~kg}$ underwent a left modified BTS with a $3.5-\mathrm{mm}$ expanded PTFE graft to relieve cyanosis caused by tetralogy of Fallot. Three days later, transient bleeding through the chest drain was noted, but it spontaneously stopped, resulting in

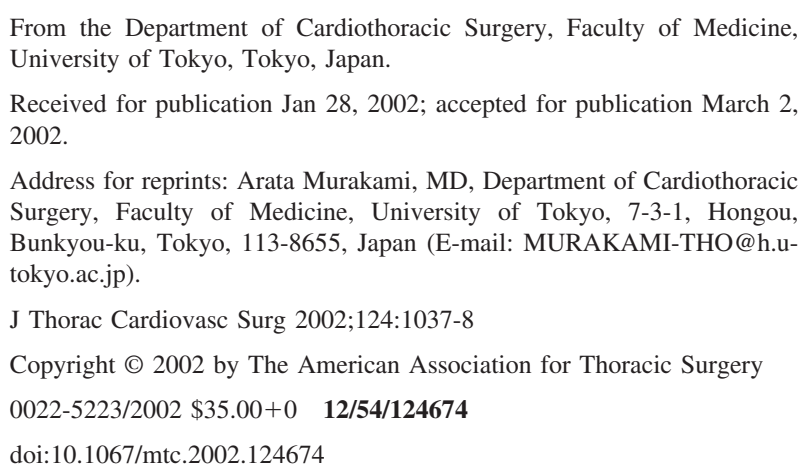

the formation of a left upper mediastinal mass. Three weeks later, growth of the mass was noticed on a chest radiograph, and ultrasonography revealed a heterogeneous mass with perigraft fluid collection. The size of the mass increased daily despite infusion of packs of frozen plasma (plasma fibrinogen level, $71 \mathrm{mg} / \mathrm{dL}$ ), and it eventually filled the upper half of the left thoracic cavity. Emergency thoracotomy was performed on the 29th postoperative day.

Reoperation revealed a gelatinous seroma $(6 \times 8 \mathrm{~cm})$ enveloping the graft. The seroma contained serous fluid around the graft. Constant serous leakage from the semitranslucent colored graft was observed (Figure 1, A) at a rate of $4 \mathrm{~g}$ in 5 minutes. Intravenous administration of fibrinogen proved ineffective.

A vascular clamp was placed at each end of the graft. The blood in the lumen of the graft was aspirated out, and $1.0 \mathrm{~mL}$ of fibrinogen-rich solution (solution A, Bolheal, $1.0 \mathrm{~mL}$ of which contains $80 \mathrm{mg}$ of human fibrinogen, $75 \mathrm{U}$ of human plasmaderived blood clotting factor XIII, and 1000 KIE of bovine aprotinin; Chemo-Sero Therapeutic Research Institute, Kumamoto, Japan) was injected through a 22-gauge needle under low pressure for 5 minutes until the solution could be seen extruding through the graft. The injection was repeated again, and the residual solution was removed by means of irrigation through a small incision in the graft. The incision was closed with 7-0 polypropylene sutures. The clamps were gently released on the pulmonary artery side first and
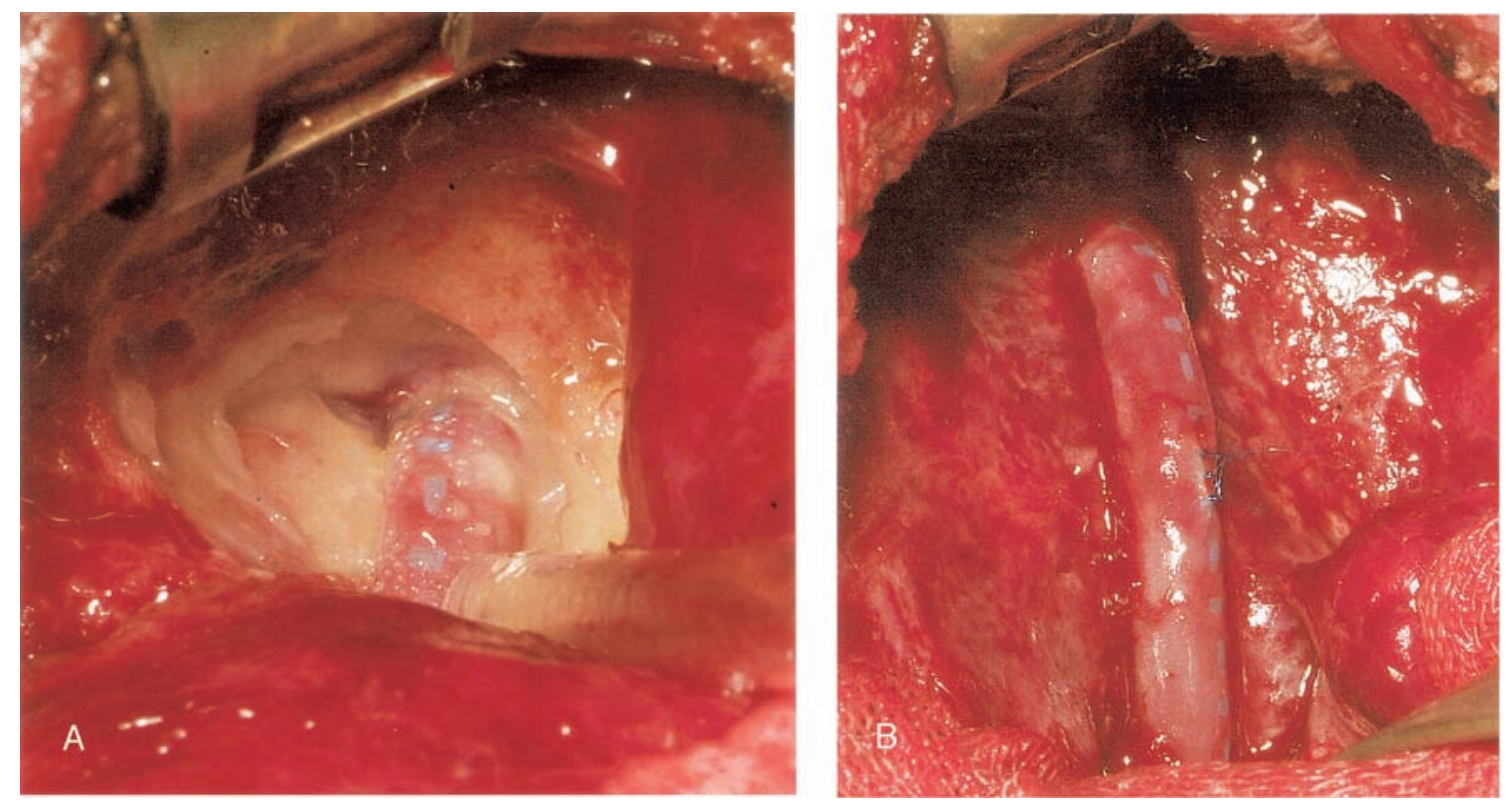

Figure 1. A, A gelatinous seroma enveloping the weeping graft. B, Serous leakage was instantaneously stopped with intraluminal fibrinogen injection. 
then the subclavian artery side. The serous leakage immediately stopped (Figure 1,B). Postoperatively, fibrinogen (heated and freeze-dried human fibrinogen; Fibrinogen HT; Yoshitomi, Inc, Osaka, Japan) was administered to maintain the serum fibrinogen level at greater than $150 \mathrm{mg} / \mathrm{dL}$, and tranexamic acid (Transamin-G; Dai-ichi, Inc, Tokyo, Japan) was administered as antifibrinolytic therapy. The patient was discharged on the 10th postoperative day. The graft was proved to be patent at the 2-month follow up.

\section{Discussion}

Excess serous fluid leakage from PTFE-modified BTSs is encountered after approximately $20 \%$ of operations, ${ }^{1,2}$ but the mechanism has not been clearly identified. Maitland and coworkers ${ }^{3}$ reported successful treatment of 2 cases of massive serous leakage by means of intraluminal injection of fibrin glue. Suzuki and associates ${ }^{4}$ reported the effectiveness of grafts pretreated with fibrinogen in preventing serous leakage and a good long-term patency rate of the pretreated grafts. We injected fibrinogen alone intraluminally to eliminate the risk of graft occlusion in a case of serous leakage and a giant seroma. Our case demonstrated that extrusion of fibrinogen through the graft has an excellent sealing effect. We speculated that the fibrinogen formed fibrin clots in the PTFE graft wall by contacting thrombin and calcium hydrochloride in the patient's own blood. We recommend this method when symptomatic serous leakage is resistant to intravenous fibrinogen administration. ${ }^{5}$

\section{References}

1. Noyez L, Daenen W. The modified polytetrafluoroethylene BlalockTaussig shunt: case report of an unusual complication. $J$ Thorac Cardiovasc Surg. 1987;94:634-5.

2. LeBlanc J, Albus R, Williams WG, et al. Serous fluid leakage: a complication following the modified Blalock-Taussig shunt. J Thorac Cardiovasc Surg. 1984;88:259-62.

3. Maitland A, Williams WG, Coles JG, Freedom RM, Trusler GA. A method of treating serous fluid leak from a polytetrafluoroethylene Blalock-Taussig shunt. J Thorac Cardiovasc Surg. 1985;90:791-3.

4. Suzuki T, Fukuda T, Katohgi T, Oda Y. Effectiveness of fibrinogen to avoid serous fluid leakage through polytetrafluoroethylene (PTFE) tubular graft. J Jpn Assoc Surg. 1990;38:596-600.

5. Hiramatsu Y, Atsumi N, Sasaki A, Mitsui T. A successful treatment of serous leakage from a polytetrafluoroethylene Blalock-Taussig shunt with intravenous fibrinogen administration. J Thorac Cardiovasc Surg. 1999;117:1230-1. 